\title{
Automatic façade modelling using point cloud data for energy-efficient retrofitting
}

\author{
M. Previtali • L. Barazzetti • R. Brumana • B. Cuca • \\ D. Oreni $\cdot$ F. Roncoroni • M. Scaioni
}

Received: 11 June 2013 / Accepted: 24 April 2014 / Published online: 10 May 2014

\begin{abstract}
Energy-efficient retrofitting of existing buildings is a key aspect for reaching the proposed energy consumption reduction targets fixed by public authorities in different countries. For this task, the availability of as-built building models is of primary importance for both diagnosis of thermal dispersion and designing of retrofitting. In this paper, we present an automated methodology to derive highly detailed $3 \mathrm{D}$ vector models of existing building façades starting from terrestrial laser scanning data. The presented methodology first accomplishes the segmentation of the point cloud of a building façade into its planar elements. Then, starting from the identified planar clusters, façade breaklines are automatically extracted to be used later to generate a $3 \mathrm{D}$ vector model. During
\end{abstract}

\footnotetext{
M. Previtali $(\bowtie) \cdot$ L. Barazzetti $\cdot$ R. Brumana $\cdot$ B. Cuca $\cdot$ D. Oreni Department of Architecture, Built Environment and Construction Engineering, Politecnico di Milano, Via Ponzio 31, 20133 Milano, Italy

e-mail: mattia.previtali@mail.polimi.it

L. Barazzetti

e-mail: luigi.barazzetti@polimi.it

R. Brumana

e-mail: raffaella.brumana@polimi.it

B. Cuca

e-mail: branka.cuca@polimi.it

D. Oreni

e-mail: daniela.oreni@polimi.it

F. Roncoroni

Polo Territoriale di Lecco, Politecnico di Milano, Via Marco

D’Oggiono 18/A, Lecco, Italy

e-mail: fabio.roncoroni@polimi.it

M. Scaioni

College of Surveying and Geo-Informatics, Tongji University, 1239 Siping Road, 200092 Shanghai, People's Republic of China e-mail: marco@tongji.edu.cn
}

this final step, some priors on urban scenes like the prevalence of straight lines and orthogonal intersections are exploited to set additional constraints. The final product is a semantically enriched 3D model of the building façade that can be integrat-ed in Building Information Model (BIM) for planned mainte-nance. Eventually, the integration between derived façade models and infrared thermography (IRT) is presented for energy efficiency evaluation of buildings and detection of thermal anomalies.

Keywords BIM · Breakline extraction · Façade segmentation $\cdot$ Laser scanning $\cdot$ Infrared thermography

\section{Introduction}

The increase in energy efficiency of existing buildings is a major task to reach the energy-saving targets fixed by public authorities in different countries. Although many factors in-fluence buildings' energy efficiency (e.g. solar exposition and building orientation, geographic location, neighbour environment, heating and cooling systems, typology of electrical appliances, and building size), one of the most important parameters is the quality of thermal insulation. In particular, the composition of the façade and roof is of primary importance because it may determine thermal bridges and heat losses from the envelop due to inefficient insulation materials. In order to properly design thermal retrofitting of such building components, highly detailed as-built models are needed on one side (i) for the thermal assessment and on the other (ii) for producing executive drawings. In particular, terrestrial laser scanning (TLS) and close-range photogrammetry are the surveying techniques mainly used for producing as-built building models. These techniques become useful as modelling tools not only in heritage applications (Alshawabkeh and Haala 2004; Boehler et al. 2002;Naci2007; Brumana et al. 2013), 
where surfaces are complex and irregular, but also for large and medium size civil structures. In particular, nowadays, an increasing interest is paid to the generation of detailed building models from laser scanning data (ElHakim et al. 2003; Lerones et al. 2010; Lubowiecka et al. 2009; Moreira et al. 2013; Oreni et al. 2012a) and in future from other emerging 3D imaging technology like time-offlight cameras (Hansard et al. 2013) and gaming devices (Menna et al. 2011). This is mainly due to the fact that automation in acquisition and registration of scans, in conjunction with a reduction of the cost of instruments, allowed to a larger number of operators the chance to use TLS.

However, raw point clouds derived from a TLS survey are generally not directly ready for practical applications. This is related to the fact that point clouds do not present any kind of topology information. In addition, they feature a low level of abstraction. Further problems rise up in correspondence of edges, corners and other features, where the spatial resolution of the point cloud is not enough to describe them. In order to overcome those problems, point clouds of buildings are generally transformed into vector format by adopting standard reverse engineering approaches. However, this task requires a largely timeconsuming manual work performed by skilled operators. For this reason, an increasing interest is paid to automatic modelling of buildings used in conjunction with (Nan et al. 2010) or as an alternative to computer-aided design (Becker and Haala 2007). Indeed, manual editing can be avoided in the case when a detailed modelling is not required or may take place only after a basic model has been automatically generated. This approach would allow an increase of the efficiency in the overall process and a cost reduction com-pared to a standard fully manual approach. Although much research work has been done, practical application to automatic modelling of full façades is still far to fall into practice. This is mainly due to the wide plethora of architectural ele-ments and styles that could be combined in façades, with large changes between different countries.

Another aspect whose relevance is rapidly growing is the semantic content of building models. Indeed, nowadays, great attention is paid to Building Information Models (BIMs). A BIM model covers more than just building geometry (Fox and Hietanen 2007; Murphy et al. 2009; Oreni et al. 2012b), being the combination of objects, relations and attributes. The use of BIMs goes beyond the design phase, extending throughout the entire building life cycle and allowing for a better planned maintenance. In addition is the possibility to add time and cost to models as further dimensions may efficiently support the decisionmaking process during the design of retrofitting works.

In the phase of assessment of the thermal efficiency of existing buildings, infrared thermography (IRT) is a powerful tool to detect the external temperature of the building envelope. Indeed, IRT can be efficiently used to detect thermal bridges and heat losses (Lagüela et al. 2011; Edis et al. 2012). For this reason, integration between BIM models and thermal images can provide new possibilities for investigators and designers.

In this paper, we present an automated procedure to generate a thermography-textured as-built 3D BIM model of a building façade for supporting energy efficiency evaluation, diagnosis of thermal anomalies, retrofitting design and control of installation work. The developed methodology starts from point cloud of building façades acquired by TLS, and it is aimed at modelling modern building where façade components are planar or piecewise planar. To this end, the unorganized point cloud of the building is firstly segmented into its planar elements (see "Façade segmentation" section). Identification of planar clusters is performed by using a modified RANSAC approach. In particular, some topology information are added in the processing in order to prevent RANS $\mathrm{AC}$ 'bad-segmentation' results reported in the literature in the case of massive point clouds (Stewart 1997). Once façade elements are identified, breaklines are automatically extracted from the point cloud (see "Breakline identification" section). In this phase, some priors on urban scenes, like the prevalence of straight lines and orthogonal intersections, are enforced. Contemporary, a further recognition and separation of detected clusters into façade elements (e.g. walls, windows and doors) is performed by using a classification tree (see "Object classification" section). Extracted breaklines and information derived from object classification are combined together to generate a 3D BIM model of the entire façade (see "BIM model generation" section). Finally, the façade model is integrated with IRT data (see "Integration of 3D facade model with thermal images" section). Indeed, the relationship between thermal data and building geometry is of primary importance in order to precisely locate surfaces affected by thermal defects.

\section{Related work}

As previously discussed, in this paper, the identification of façade elements is performed by means of segmentation of the whole point cloud into planar clusters. Point cloud segmentation can be defined as the process aimed at grouping points sharing a similarity on their spatial properties or related to some attributes (intensity, colour or others). Segmentation techniques were initially developed for the analysis of airborne laser scanning (ALS) data (Masaharu and Hasegawa 2000; Geibel and Stilla 2000). Then, some of those algorithms proved their suitability also to TLS for façade analysis (Lerma and Biosca 2005; Pu and Vosselman 2006; Stamos et al. 2006). Segmentation methods presented in the literature can be classified into three main groups: (i) region growing, (ii) clustering of features, and (iii) model fitting. Region growing 
methods relay on the main assumption that neighbouring point in the cloud presents similar characteristics. For this reason, those methods are based on the aggregation of homogenous points starting from a small set of seed points. For example, in the case of plane identification (Pu and Vosselman 2006), a seed with its nearby points is randomly chosen in the cloud. If a plane fits these points within a user-defined threshold, the surface is accepted and the growing procedure starts. The growing phase, that is the extension of the detected plane to nearby points, is based on a similarity criteria which can be point proximity, global planarity or point normal vector direction. The growing process stops when newer points do not fit any more the user-defined threshold for similarity. Generally, region growing methods suffers from the correct identification of a seed point and noise in the point cloud. Indeed, an initial wrong selection might lead to wrong segmentation results. Clustering of features methods (Filin 2004; Hesami et al. 2010; Martínez et al. 2012) can be seen as a combination of two processes: first, patterns in the data are identified on the basis of some attributes, then they are clustered based on them. Even if this method is quite general, identification of proper features may be complex, and segmentation results are highly affected by this selection. In addition, clustering multidimen-sional features can be computationally infeasible for large point clouds. Model fitting methods (Schnabel et al. 2007; Boulaassal et al. 2008) are based on the identification of geometrical primitives in the point cloud. Points conforming to a defined primitive are grouped as belonging to the same cluster. Model fitting methods mainly relies either on RANS AC (Fischler and Bolles 1981) or on Hough transform (Hough 1962) in order to find primitives into a noisy dataset. In particular, RANSAC showed a higher efficiency in comparison with the Hough transform in the case of TLS data (Tarsha-Kurdi et al. 2008). However, RANSAC is just an algorithm measuring the consensus to a certain assumed model. Consequently, the extracted primitives may not correspond to real architectonical object. This limit is well documented in literature as badsegmentation (Stewart 1997). In particular, bad-segmentation results may be categorized into the following:

- under-segmentation, in the case when several features are segmented into one; and

- over-segmentation, when one feature is segmented into several ones.

Although the segmentation problem has been widely covered in the literature since different years, only recently, the goal of using segmentation results for producing parameterized high-level models emerged. In particular, two different approaches were proposed: interactive modelling and fully automated processes. In Nan et al. (2010), an interactive tool for assembling architectonical models directly on the point cloud is presented. In a first step, the user defines some simple architectonical elements which are then snapped to similar objects in the cloud. In a similar way, in Zheng et al. (2010), an interactive method for the completion of holes in laser scans of building façades is presented. However, in the case of interactive modelling, an extended processing time and long human intervention are needed. A full automated processing is presented in Pu and Vosselman (2009), where segmented TLS data are fitted with rectangular shapes to generate polygonal façade models. Recently, Vanegas et al. (2012) proposed an automated approach based on 'Manhattan World' building geometry assumption (Coughlan and Yuille 2001). This system classifies data into features which are then grouped in connected clusters. Finally, a volumetric model of the building is generated. For a complete review about this topic, we address to Musialski et al. (2012).

The classification of façade elements extracted from point cloud data is also a topic addressed by different works in the literature. Most of them exploit on some priors for carrying out the classification (Ripperda and Brenner 2006; Becker 2009). In particular, some specific grammar rules are used for the description of such repetitive patterns like the regular distribution of windows and doors in building facades. An automatic extraction and classification algorithm of building features is also presented in Pu and Vosselman (2009). In this case, objects derived from segmentation are classified according to some parameters like their position and extent.

Because the main issue for an accurate integration of thermographic images and building models is the registration of scans and thermal images in the same reference system, many applications focus on this topic. In many cases, thermal images are processed individually by using homographic transformations (González-Jorge et al. 2012). However, this method is rigorous only in the case of planar façades. For this reason, a more robust method is presented in Cabrelles et al. (2009), which made use of space resection for the rigorous orientation of single images. This approach has been then extended to work with the contemporary registration (bundle adjustment, see Luhmann et al. (2011)) of several thermal images integrating a photogrammetric block of red-greenblue (RGB) images captured by using a consumer-grade calibrated single-lens reflex (SLR) camera (Barazzetti et al. 2013). In order to speed up the whole process, some ad hoc acquisition systems were also designed. In Alba et al. (2011), a 'bi-camera' system, coupling a traditional RGB camera with an infrared (IR) sensor, was proposed. In this case, a standard RGB camera and a thermal camera were rigidly coupled together. In the processing phase, only RGB images were oriented with a standard bundle adjustment deriving their exterior orientations. Then, thanks to the knowledge of the relative orientation between RGB and IR cameras, determined in a calibration phase (Luhmann et al. 2010; Yastikli and Guler 2013), thermal images are oriented. 
The façade modelling procedure and thermal image integration

In this paper, we present a novel methodology for automatic façade modelling. In particular, the procedure follows a scheme that goes from scan acquisition up to point cloud segmentation, automatic breakline extraction, object classification and generation of the final 3D BIM façade model and eventually its integration with IR images. Each step of the procedure presents some advances with respect to the state of art.

In this paper, we addressed to modern building where all the elements constituting the façade can be modelled as planar objects or at least can be approximately divided into piecewise planar parts. Modelling of complex historical buildings where constituting elements (e.g. columns and vaults) are properly represented by more complex geometrical primitives (e.g. cylinders and cones) and façades are abundant of decorative elements is not addressed in this work.

The developed modelling methodology can be applied to unstructured point cloud of tens of millions points. This means that each point is parameterized by its spatial coordinates and may also feature some related attribute (e.g. intensity or colour) but does not share any topological relationships with the points in its nearby. The input point cloud can be generated by a single or multiple laser scan station(s). Indeed, after scan registration/geo-referencing, scans are merged together without needing any reorganization into a specific data structure. The overall procedure is presented in Fig. 1. Once all scans are acquired and registered together, the main elements constituting the façade are firstly identified by means of a segmentation process based on a modified RANSAC implementation. In particular, the standard RANSAC approach for point cloud segmentation is modified by adding the topology in the process. This topological information between scan points and detected clusters is added in order to minimize problems connected to under- and over-segmentation, respectively.

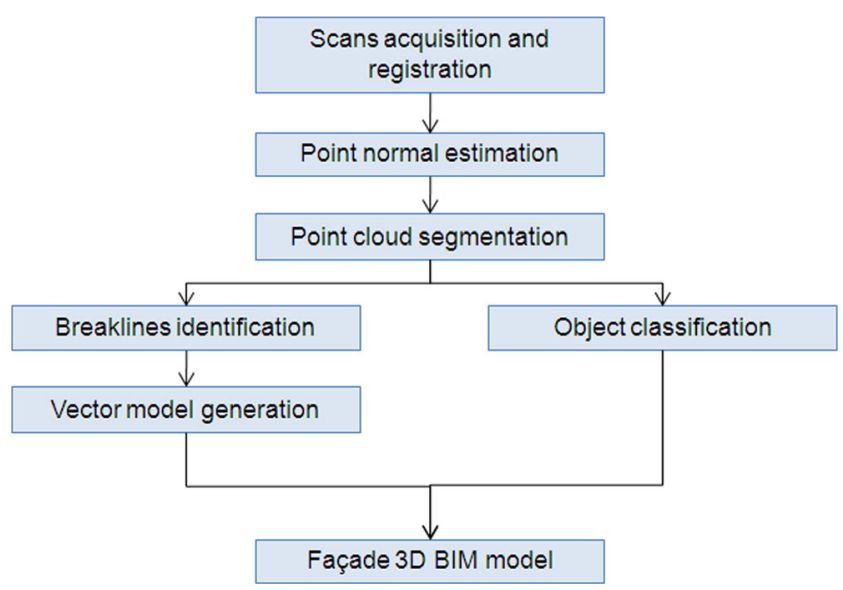

Fig. 1 Pipeline of the facade modelling process
Once planar clusters constituting the façade are detected, façade breaklines are automatically derived. During this phase, some constraints related to façade geometry, like the prevalence of straight lines and orthogonal intersections, are enforced to obtain a regularization effect. At the same time, a further classification is performed, on the basis of some priors on the façade structure organized in a classification tree. In this way, detected objects are classified into façade elements (e.g. walls and windows). Detected breaklines are then used to obtain the 3D geometry of the façade while classification results are used to enrich geometrical data with semantics and to generate a $3 \mathrm{D}$ BIM model of the entire façade. Finally, the integration between thermal data and geometric building model is obtained by mapping thermal images on the 3D semantically enriched model of the building. Main steps of processing IRT data are reported in the following subsections, while more details can be found in Previtali et al. (2013).

A remark is needed to the fact that several algorithms presented in this work rely on the extraction of normal vector information at the point locations, and usually, scanning devices do not directly provide that. For this reason, point normal estimation is performed using the approach proposed by Jenke et al. (2008). For each point in the cloud, the ten nearest neighbours are considered and a plane is fitted. Afterwards, the number of nearest neighbours is increased iteratively until a stable solution for the normal vector is found.

\section{Façade segmentation}

The first step in our approach is the segmentation of the raw point cloud into planar elements constituting the building façade. Indeed, as previously discussed, in the case of modern buildings, the majority of façade components are planar. The detection of other shapes is not relevant for buildings' styles analysed in this paper.

Detection of façade elements is performed by means of a modified RANSAC approach (Fig. 2). In particular, the de-veloped approach works in a sequential manner. Indeed, at the each iteration, the plane representing the maximum consensus in term of points according to the RANSAC principle is detected (generally, it is the largest plane in the cloud). Then, points which are compatible with this plane (inliers) are removed and, in the next iteration, a new one is detected. The process stops when the size of the maximum consensus plane detected is lower than the minimal size defined by the user. However, two major shortcomings reported in the literature about the sequential application of RANSAC algorithm for point cloud segmentation are the following:

- its relative inefficiency in terms of computational time; and

- the detection of spurious planes. 
Fig. 2 Workflow of the facade segmentation process

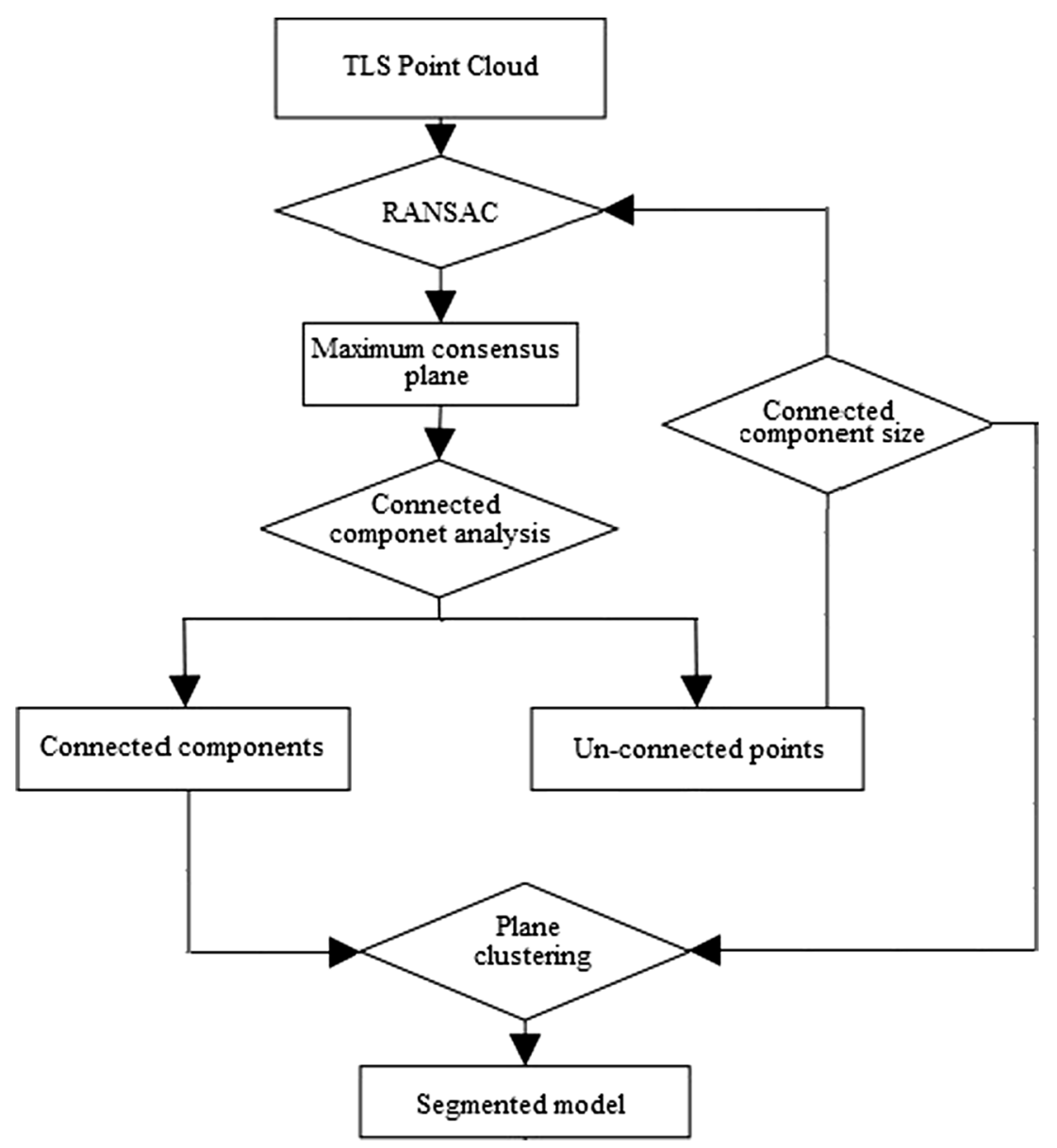

In this paper, we will not address to RANSAC variants designed to increase computational efficiency. We only used the adaptive approach proposed by Hartley and Ziesserman (2003). This modified version of RANSAC may determine in adaptive way the number of samples to be tested according to the sample size and the number of inliers. In this way, the processing time can be significantly reduced if compared to the standard approach.

A major effort was made to reduce bad-segmentation problems reported by several segmentation algorithms (Awwad et al. 2010; Boulaassal et al. 2009;PuandVosselman2006; Sapkota 2008). As previously mentioned, bad-segmentation results can be categorized into 'under-segmentation' and 'over-segmentation'. Undersegmentation is generally due to the fact that points constituting the maximum consensus to RANSAC in reality belong to different objects. A typical example is represented by windows. Indeed, even if they belong to the same geometrical plane, each window represents a distinct architectonical component. On the other hand, oversegmentation is generally associated with noise or irregularities in the data. Indeed, many façades present several irregularities like out of plumbs and variation of shapes that are not evaluated in the RANSAC inlier estimation. For example, in the case of a façade presenting an out of plumb, the segmentation may result in subdividing a single façade wall into several objects.

In order to minimize under-segmentation problems, two different strategies were included in the developed segmentation pipeline. The first one checks the consistency of point normal vectors and the estimated RANSAC plane normal. This strategy is designed to prevent clustering together points belonging to the same geometrical planes but having different orientations. The consistency check is performed during the RANSAC inlier evaluation. Indeed, once three points are sampled from the cloud and a plane is estimated, inlier evaluation takes into consideration not only the distance from the estimated plane but also the angular distance between point normal and plane normal.

In Fig. 3a, the effectiveness of this check is shown. In the case, this consistency check is not performed; those points highlighted in red are clustered with the façade wall while they belong to the building roof. The evaluation of the direction of the local point normal helps reduce these problems. The second strategy copes with the problem of different objects having the same direction and belonging to the same geometrical plane (the problem of windows as previously discussed). In this case, information about point topology is introduced. Points are not usually related by any topological relationship 
a.

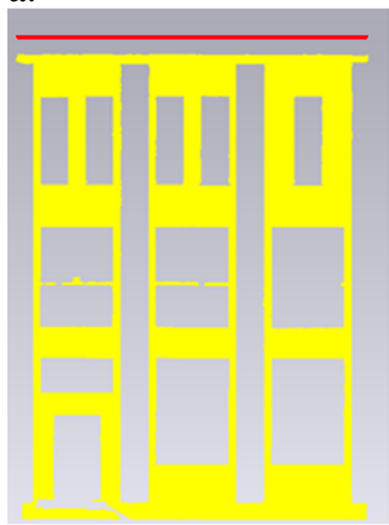

b.

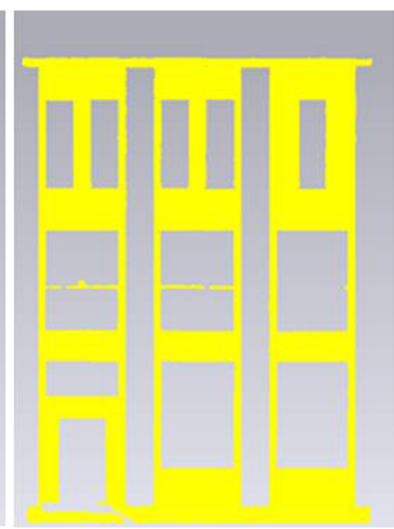

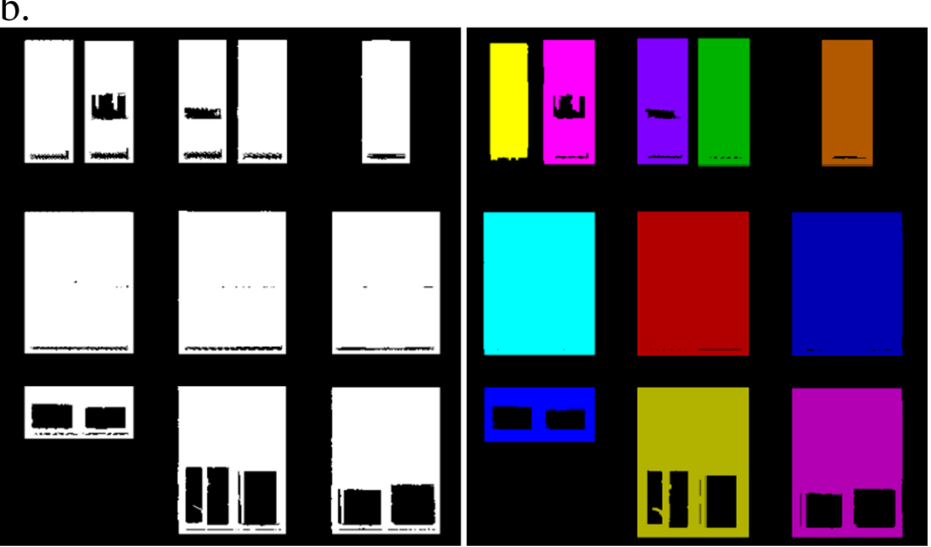

Fig. 3 Under-segmentation removal: a segmentation results without (left) and with point vector consistency check; $\mathbf{b}$ bitmap for the window cluster (left) and detected connected component represented with different colours (right)

in a point cloud but, if their density is sufficiently high to sample in non-ambiguous way the surface, we can assume that points belonging to the same object should be sufficiently close to each other while groups of points belonging to different objects should have a gap between them. For this reason, once a maximum consensus plane is defined, the connectivity between points is valuated. This connectivity check is performed by generating a raster bitmap. In particular, the bitmap is obtained by projecting each point along the normal direction to the plane. If a cell of the bitmap is occupied by at least one inlier, its value is set to 1 , otherwise it remains equal to 0 (Fig. $3 \mathrm{~b}$ ). The cell size in the bitmap is chosen as the mean sampling resolution in the point cloud. Indeed, larger cells may sample in a too coarse way the point cloud and do not overcome the under-segmentation problem. On the other hand, a too small cell size may feat the limit of the scan ground sampling distance (GSD). Once the bitmap is setup, cells representing a connected component can be easily found. Then, all points whose projection belongs to the same connected component are grouped into the same cluster (Fig. 3b). In this phase, some spurious segments might be found. Indeed, each segment whose area is lower than the user-defined minimal size is rejected.

Over-segmentation problems can be prevented by increas-ing the RANSAC tolerances. However, this may lead to 'under-segmented' parts which may be difficult to split again. For this reason, in our work, we keep the RANSAC tolerance low and a moderate over-segmentation is preferred. Indeed, when the RANSAC plane detection ends, over-segmented parts are combined together in a clustering phase (Fig. 4). In particular, three parameters are evaluated in the clustering: (i) similarity of normal vectors, (ii) perpendicular distance be-tween planes, and (iii) intersection between planes. First, located objects are clustered by using the mean shift algorithm (Comaniciu and Meer 2002), according to their normal vectors. Then, into each family of planes, the perpendicular distance between clusters of points classified as representing different objects is evaluated. If this distance is lower than the user-defined RANSAC threshold and the convex hulls of the two point clusters intersects, they are recognized as a single object and fused together.

The developed segmentation methodology was tested on a building of Politecnico di Milano universityCampus D'Oggiono (Fig. 5a). This dataset was acquired by a Faro Focus 3D laser scanner. Some technical specifications of this laser are presented in Table 1.

In this case, three scans of the building façade were performed and registered together by using 14 checkerboard targets whose centre was also measured with a first-order theodolite Leica TS30. Statistics shows a registration precision in the order of $2 \mathrm{~mm}$. After registration, scans were merged together into a unique point cloud of about 15 million points having a GSD of $5 \mathrm{~mm}$. This point cloud was segment-ed by using the presented approach into 120 planar clusters (Fig. 5b).

\section{Breakline identification}

Once the façade has been segmented into planar clusters, breaklines are automatically identified in order to derive a high level of detail vector models of the building. To this aim, a contour extraction algorithm enforcing urban scene priors has been developed. This algorithm relies on the observation (Boulaassal et al. 2009) that points belonging to a cluster contour can be individuated as extremities of long sides of Delaunay triangles. For this reason, as a preliminary step, a new coordinate system for each planar cluster is defined performing principal component analysis (PCA). The original coordinates $\left(X_{\text {or. }}, Y_{\text {or., }} Z_{\text {or. }}\right)$ are then transformed in a new local space aligned along the principal component directions $\left(X_{\text {new }}, Y_{\text {new }}, Z_{\text {new }}\right)$. In particular, the component $Z_{\text {new }}$ which is approximately directed along the normal direction to the planar cluster may be considered as negligible. Once the new coordinates are calculated $\left(X_{\text {new }}\right.$, $Y_{\text {new }}$ ), a bi-dimensional 
Fig. 4 Over-segmentation removal: a a small out of plumb $(1 \mathrm{~cm})$ is detected in the façade (green cluster); b clusters are merged after clustering all planes a.

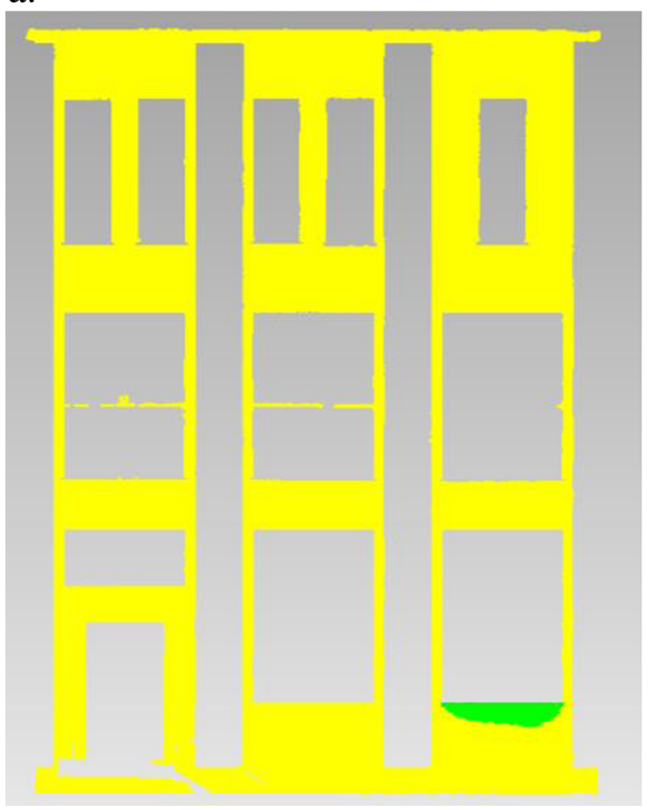

b.

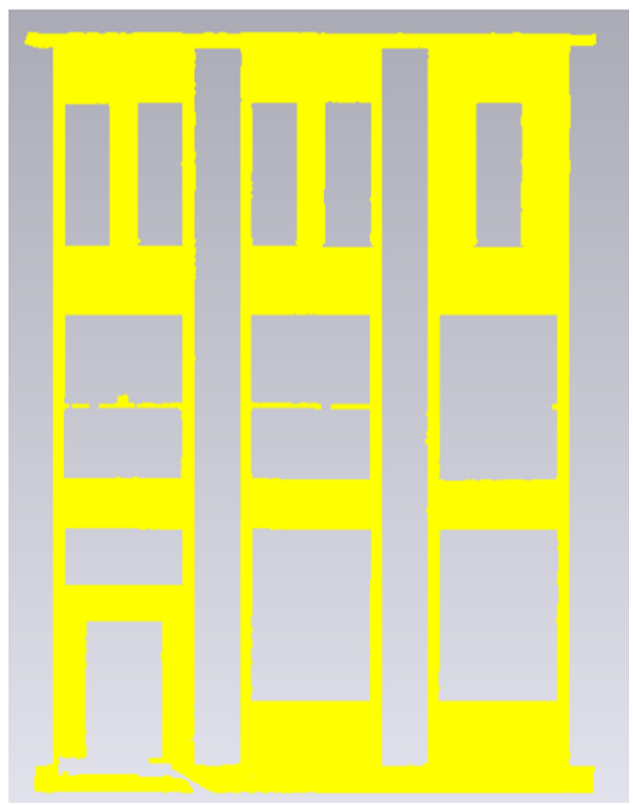

Delaunay triangulation is performed and contour points of each clusters are individuated at the extremities of long-side triangles (Fig. 6a). Even if this method is quite robust in the extraction of contours, the results obtained in this way (Alshawa et al. 2009; Boulaassal et al. 2007; Martínez et al. 2012) are quite irregular showing a characteristic 'sawtooth' shape as can be seen in Fig. 6b. However, this is in contrast with the characteristic façade geometry where straight lines are predominant, and they are mainly horizontal or vertical. For this reason, in order to prevent this irregular edge effect, a new approach for edge regularization and breakline extraction has been developed based on a straight line and a surface intersection constraint. Once the contour points are determined, the dominant edge directions are identified by using a parsing implementation aimed at detecting dominant lines. In the case where nearly horizontal or vertical directions are detected in the contour, a horizontal or vertical constraint is enforced (Fig. 6c). Finally, edges are transposed in the original datum.

However, extracted edges do not represent the final façade breaklines yet. Indeed, spatial resolution of the point cloud is generally not enough to describe breaklines in a proper way. In addition, due to the use of RANSAC, in the case of
Fig. 5 Raw point cloud of a building façade (a); segmented point cloud(b), where each segment is represented with a different colour a.

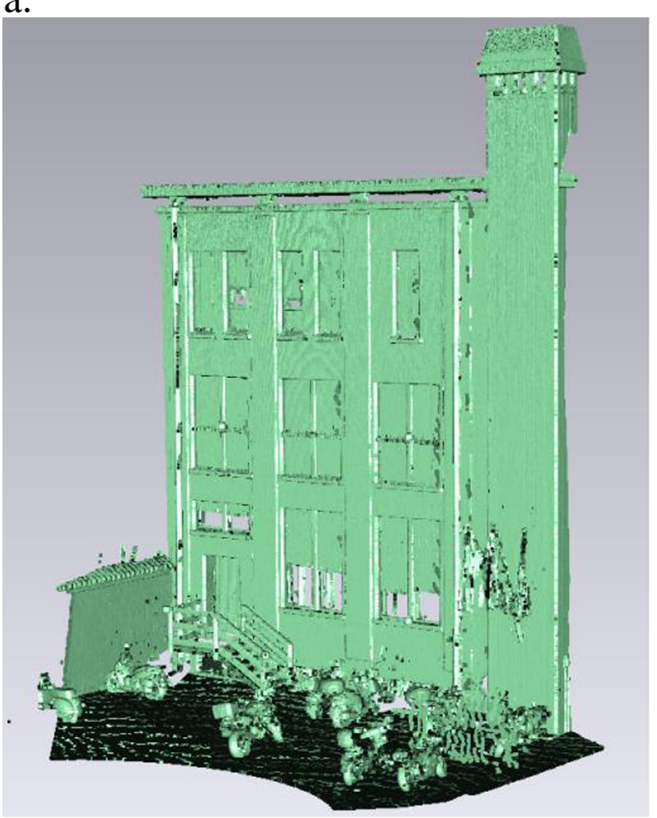

b.

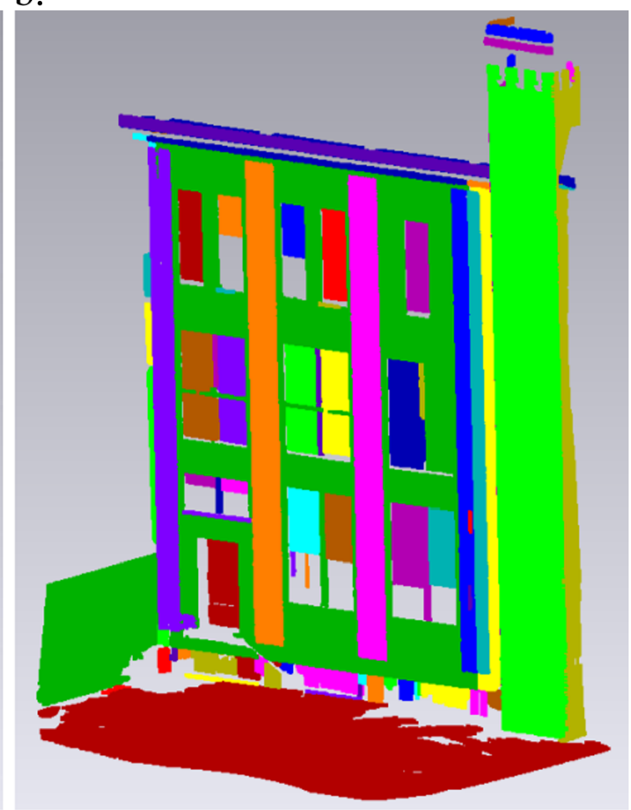


Table 1 Technical specifications of Faro Focus 3D laser scanner

Faro Focus 3D technical specifications

\begin{tabular}{ll}
\hline Range measurement & Phase shift \\
Range for distance & $0.6-150 \mathrm{~m}$ \\
Angular resolution & $0.009^{\circ}$ \\
Measurement speed & $120.000-976.000$ points/s \\
Precision at $10 \mathrm{~m}$ & $0.6 \mathrm{~mm}(90 \%$ reflectivity $)$ \\
& $1.2 \mathrm{~mm}(10 \%$ reflectivity $)$ \\
Precision at $25 \mathrm{~m}$ & $0.95 \mathrm{~mm}(90 \%$ reflectivity $)$ \\
& $2.2 \mathrm{~mm}(10 \%$ reflectivity $)$ \\
\hline
\end{tabular}

intersecting surfaces, the points lying on the intersection (that belong to both surfaces) are assigned only to one cluster (generally to the larger one). Due to these shortcomings, edges of the intersecting surfaces do not coincide anymore (Fig. 7a). In order to enforce the surface intersection, nearly identical edges are detected. In particular, two edges are classified as nearly identical if the distance between them is lower than the RANSAC threshold used for plane detection. Once two nearly identical edges are detected, surface intersection is enforced (Fig. 7b). Surface intersection constraint not only reestablishes topology between objects but also increases accuracy of detected breaklines. Indeed, by means of surface intersection constraints, breaklines are calculated as intersection of planes which are estimated from a large set of points. On the other hand, edge calculation from contour points may be more affected by noise in the point cloud. In addition, as previously discussed, the accuracy of laser scanning measure-ments in correspondence of edges is generally lower than the one on smooth surfaces.

Finally, detected breaklines are exported into a $D X F$ (drawing exchange format) file that can be loaded into a CAD environment for manual editing and drawing production (Fig. 8a). However, for many applications, a simple edge representation is not sufficient and a more complex surface model is needed (Fig. 8b). In those cases (like in BIM), a constrained Delaunay triangulation, where façade breaklines represent constraints, is used.

\section{Object classification}

The segmentation and the identification of breaklines both lack of semantic understanding of each object of the façade. However, for many applications, and in particular in the case of thermal retrofitting, an enriched façade description is of primary importance for design purposes. Consequently, the derived façade model needs of undergoing a semantic classification.

In a façade, each feature has some peculiar characteristics which can be described as recognition rules that can be derived either from the statistical analysis of a training set, or they can be based on the knowledge of some priors on the façade and urban scene. The former approach has the main disadvantage of an
Fig. 6 Edge extraction: Delaunay triangulation (a) and boundary edges before (b) and after (c) the straight line constraint application

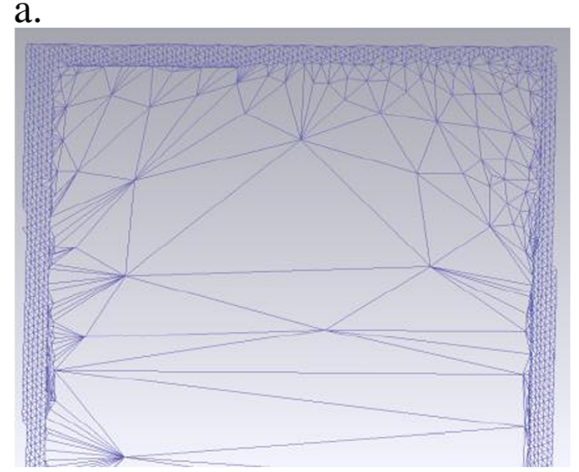

b.

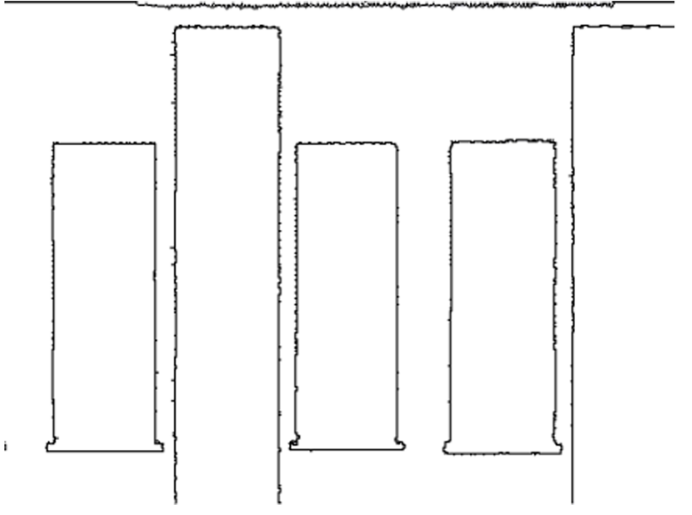

c.

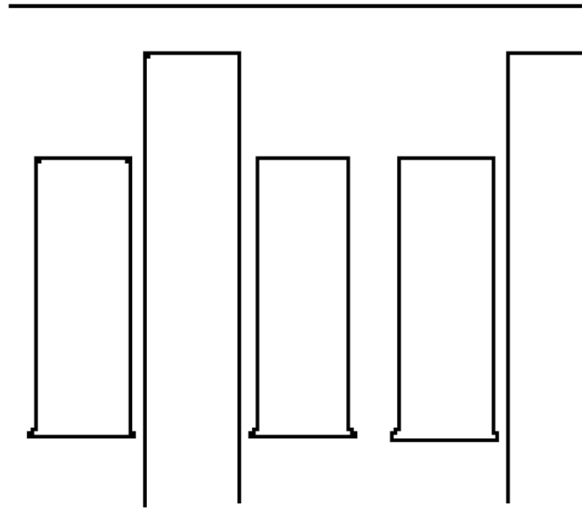


Fig. 7 Plane connection before (a) and after (b) the surface intersection constraint a.

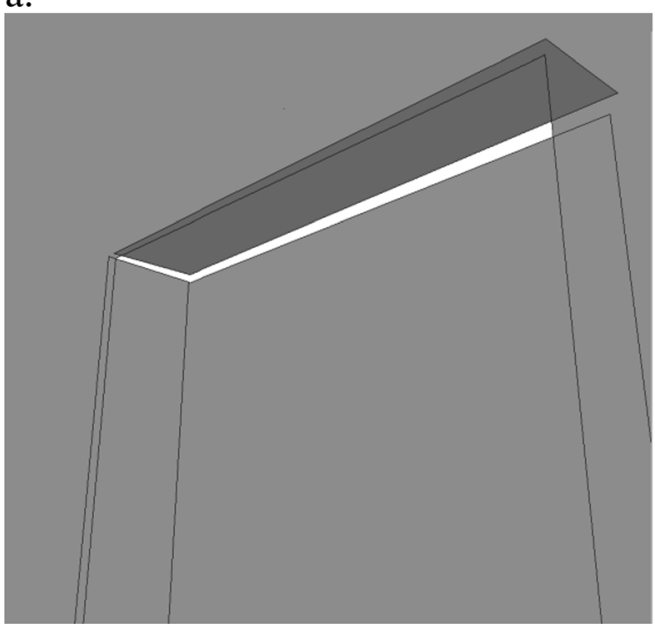

b.

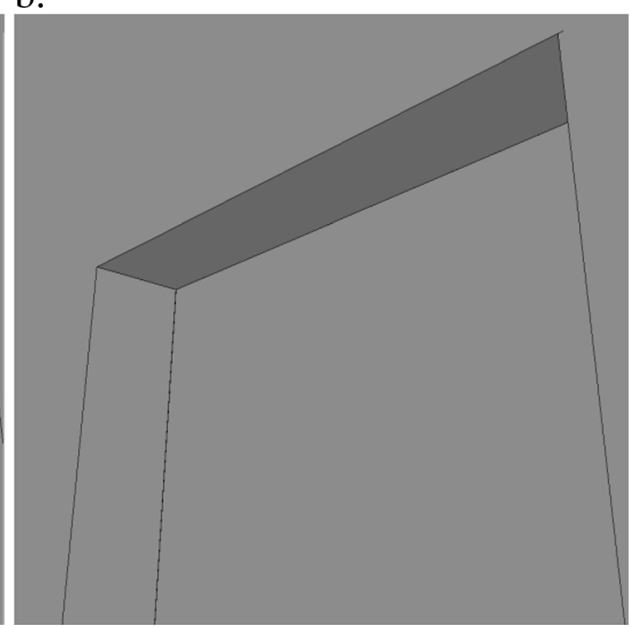

out-of-core training phase based on a wide number of manually classified cases. However, they can be generally applied for a wide variety of building types. The latter is applicable only for the building types featuring the defined priors. The classification presented in this paper belongs to this second group. On the other hand, to increase the field of application, the defined rules are quite general, and they can be used for a wide variety of building types. Only for a limited number of architectural styles are the defined priors no more adequate. In particular, the following characteristics are evaluated:

- Object size: for example, façade walls can be easily distinguished by other elements because they have a larger area;

- Object position and topology: for example, assuming that doors can be only at the bottom floor, roofs are always on the top of walls and the ground is the object at the lowest level; and

- Object orientation: for example, we assume that walls are vertical, while ground and roofs are horizontal or mildly sloped.

The recognition rules used for different object characteristics are summarized in a classification tree (Fig. 9) which is used to drive the entire process.

The classification process begins with evaluating both area and position of all the detected objects. First, the ground is detected because it is the horizontal (or pseudo-horizontal) object at the lower level. Then, façade walls are extracted since they are perpendicular to the ground and with the largest area with respect to all other vertical objects. Indeed, the wall area in a façade is generally much larger than the one covered
Fig. 8 Detected breaklines (a) and reconstructed building geometry (b) a.

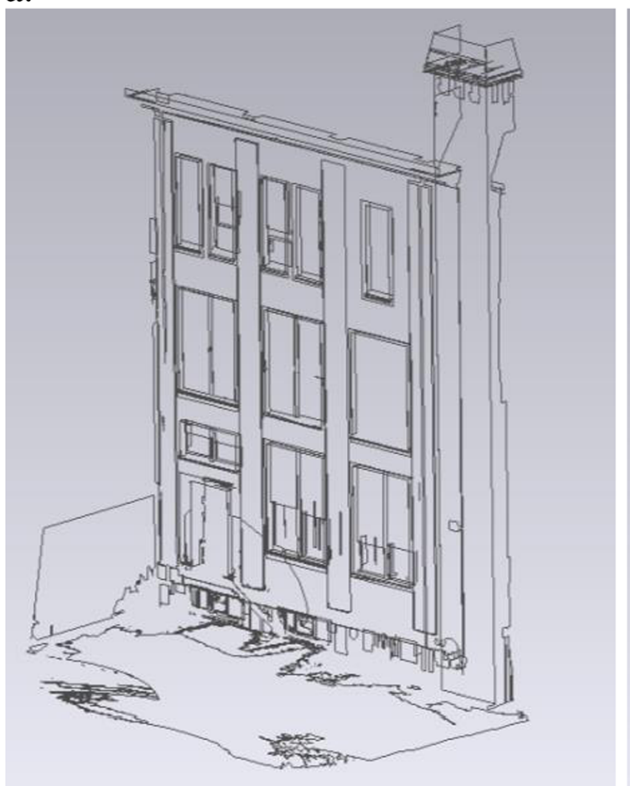

b.

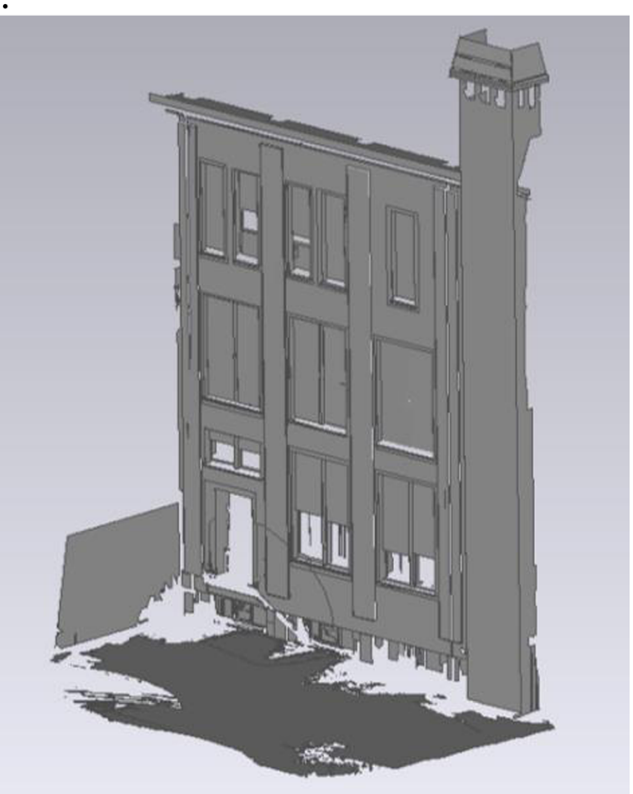




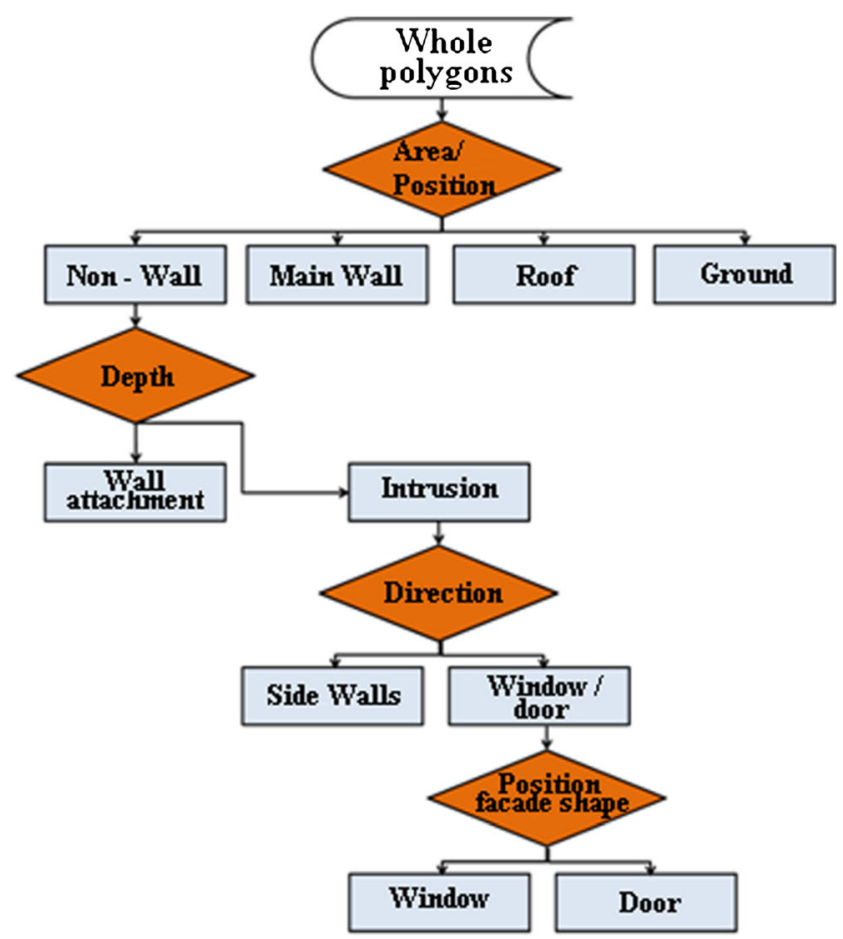

Fig. 9 Classification tree. Orange diamonds are conditions while blue rectangles represent facade elements

by other vertical objects, like windows or doors. In addition, main façade planes are generally less than sidewalls, windows and doors, so they can be considered as outliers and can be easily detected by robust statistics. Flat-like objects over walls and protruding out from the façade are classified as roof. For all non-classified objects, their position with respect to the main façade wall is evaluated. Objects in front of the façade are considered as extrusion objects and then classified in a general way as wall attachments. Objects beyond the plane of the façade are first assigned as intrusions, then split into sidewalls, windows and doors. Sidewalls can be easily recognized because, in contrast to other intrusions, their orientation is perpendicular to façade walls. In order to distinguish between doors and windows, it is assumed that doors are only at the bottom floor. In addition, in correspondence of a door, the main façade plan has a characteristic gap having an inverted ' $U$ shape', while in correspondence of windows, the gap has an 'O shape'. Starting from these considerations, doors are searched only at the ground floor and in correspondence of inverted ' $U$ shape' gaps in the main wall face. Other intrusions parallel to the façade plane are classified as windows.

BIM model generation

Even if several geometric file models have been developed in both computer graphic and CAD fields, files allowing semantic definition are relatively few. The two most prominent standards are Industry Foundation Classes (IFC) and City Geography Markup Language (CityGML) (Isikdag and Zlatanova 2009). Even if the two data structures share some similarities, several works in the literature (Benner et al. 2005; Isikdag and Zlatanova 2009; Nagel et al. 2009) showed differences between these two standards both in the geometry description and in semantic object definition. In particular, IFC holds a more detailed information about building objects than CityGML. For this reason, no straightforward translation rules between IFC and GityGML standards are available. For this reason, once the building geometry has been defined (see "Breakline identification" section) and each façade objects has been classified (see "Object classification" section), the 3D model of the building façade is generated both in CityGML and IFC standards, in order to allow high interoperability with several CAD environments as well as thermal evaluation performance software packages.

CityGML (Gröger and Plümer 2012) is a metalanguage (Fig. 10), developed starting from the schema of the Geography Markup Language 3 (GML3) and the XML file format, for the representation of 3D urban objects, and has been recently adopted as an international standard for modelling cities in the Open Geospatial Consortium (OGC) and the EU. The CityGML language defines classes and relations for the most relevant objects in cities with respect to their geometrical, topological and semantic properties. An important aspect is that it is applicable both for large regions and small areas and can represent $3 \mathrm{D}$ objects in different levels of detail. In particular, four levels of detail (LOD) were defined in order to represent buildings:

- LOD1: buildings are represented by block models with flat roofs;

- LOD2: buildings are represented by blocks but have a differentiated roof structure;

- LOD3: denotes architectural models with detailed wall and roof structures, balconies and windows; highresolution textures can be mapped onto these structures; and

- LOD4: completes a LOD3 model by adding interior structures for 3D objects (e.g. rooms, interior, stairs, and furniture).

Nowadays, IFC is seen as the strongest player of the BIM world. IFC is an object-oriented format developed by the International Alliance for Interoperability (IAI). The goal of IFC is to specify a common language for building industry technology aimed at improving 

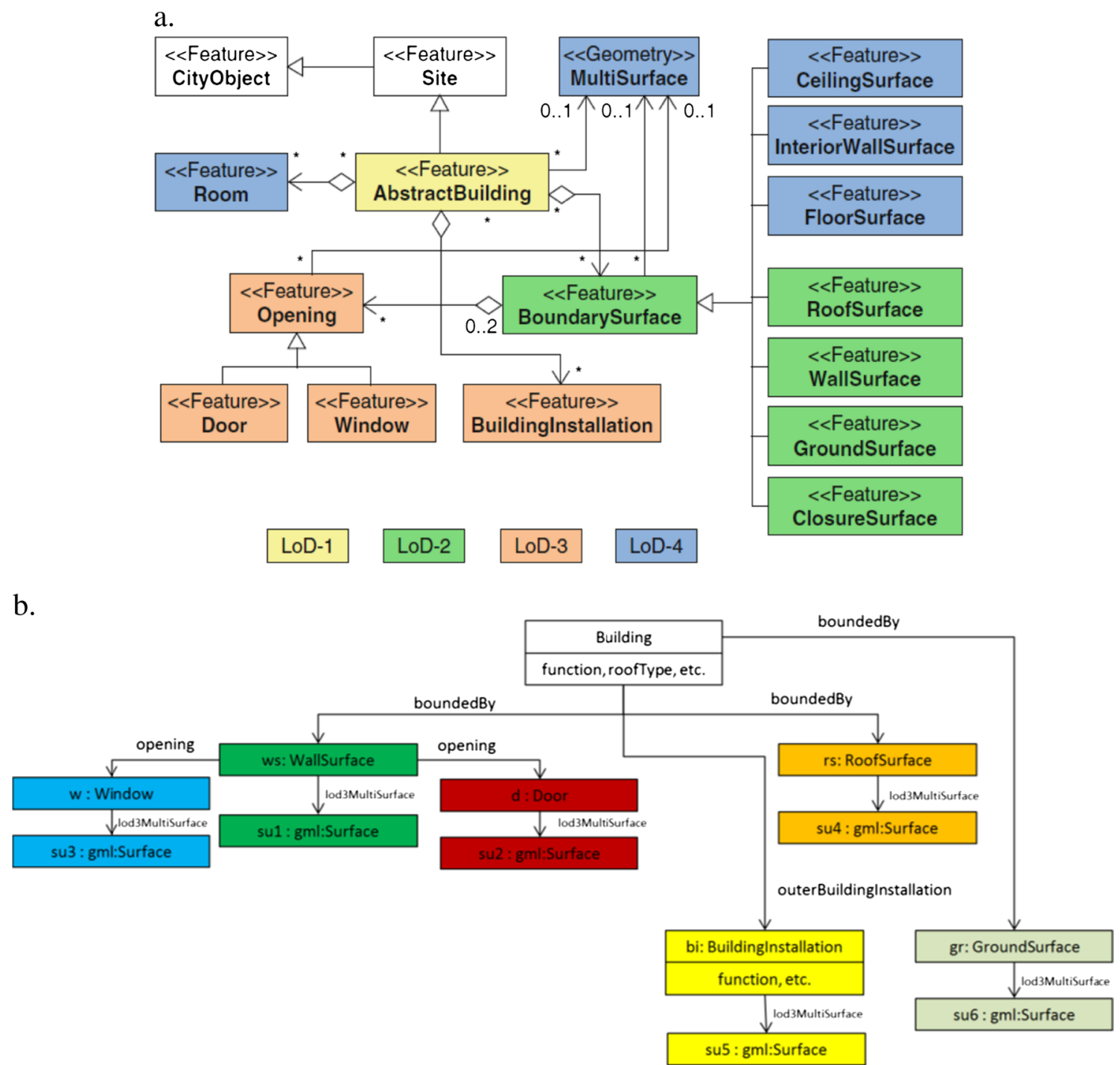

Fig. 10 CityGML file structure at different LODs (a) and feature structure at LOD3 showed as UML instance diagram

communication, productivity, delivery time, cost, and quality throughout the design, construction and maintenance life cycle of buildings (Hallberg and Tarandi 2009). Each specification (called 'class') is used to describe a range of things that have common characteristics. In particular, building elements and opening elements are subtypes of structural element. Each building element has zero or more opening elements, i.e. a wall without any door or window has zero openings, whereas each opening element (like a door and window) is attached to only one building element. Figure 11 shows 12 types of building elements that can represent a building structure in IFC standard.

The object features derived from the previous classification step can be used in a straightforward way to generate a CityGML model at LOD3 (Fig. 12a). Indeed, the features defined in CityGML meta-language as RoofSurface, WallSurface, GroundSurface, Window,
Door, BuildingInstallation, andGroundSurface have a clear correspondence with previously classified objects. This standard has a high flexibility. An important aspect is given by the fact that additional information can be addedtothe model,e.g.theLocation which indicates the global position of the building and its orientation. This can be used to evaluate façade exposition and sunlight or other descriptive data which are of major interest for energy efficiency evaluation, like the insulation value ( $U$ value) for windows, walls, floors and roofs (see Africani et al. (2013)).

However, the previously defined classification appears too coarse for the IFC standard. Indeed, objects that were previously classified in a general way as wall attachments in IFC files are classified as different features (e.g. IfcStair and IfcRailing). This is the limiting aspect underlined by different authors for direct conversion between CityGML and IFC. However, even if the concept of LOD is not 
Fig. 11 Simplified IFC file structure

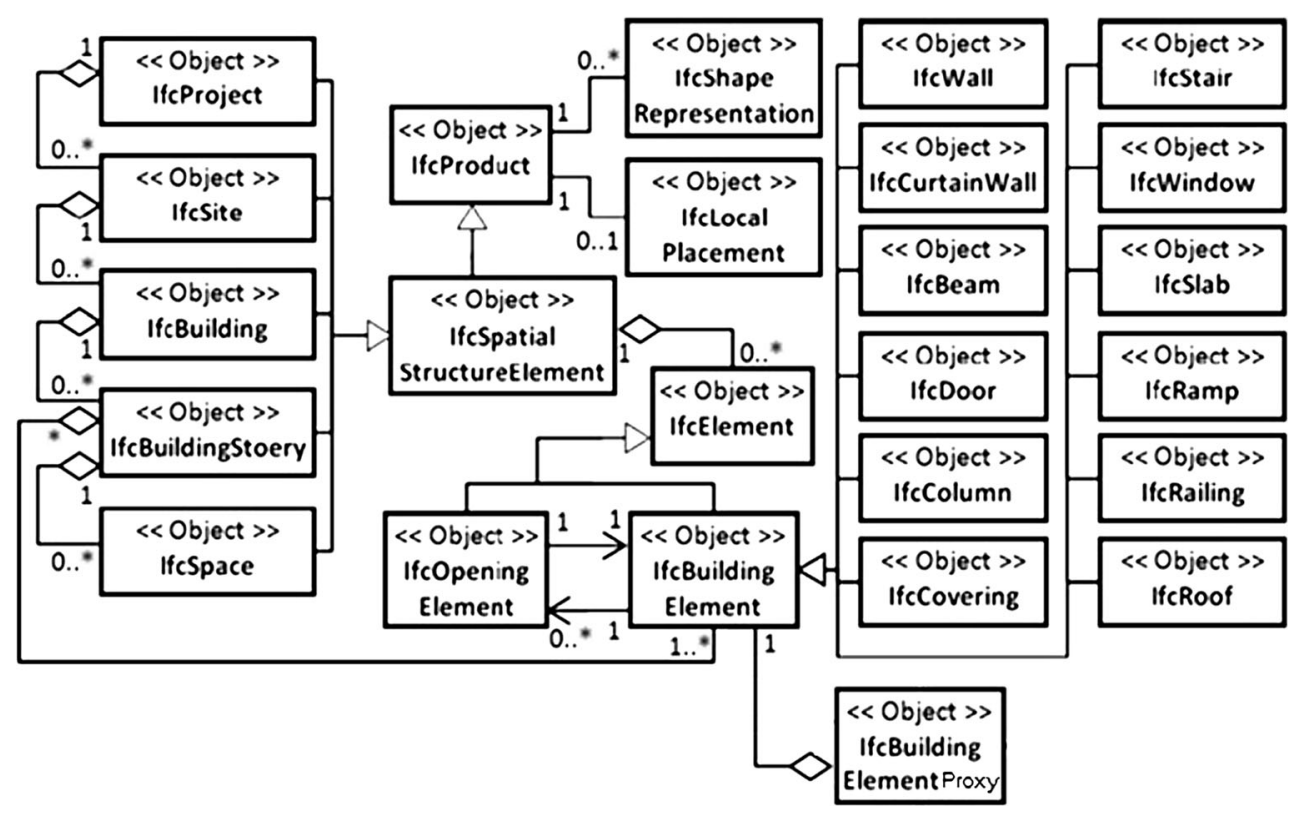

directly related to the IFC data structure, different levels of details can be also be introduced in the modelling of a building in BIM environment both in the geometrical representation and in the semantic definition. For this reason, considering a LOD lower than the maximum one allowed by IFC standard, different semantic objects can be grouped in the same IFC class. In particular, an object classified as a wall attachment can be represented in the IFC standard as IfcBuildingElementProxy; i.e. a definition that provides the same functional ity as an Ifc Building Element, but without having a defined meaning of the special type of building element it represents. In a similar way, the ground can be represented in IFC as a particular kind of IfcSlab that can be defined for example

Fig. 12 The CityGML (LOD3) façade model (a) and the same façade represented in IFC standard (b) present different semantic classification due to the differences between standards
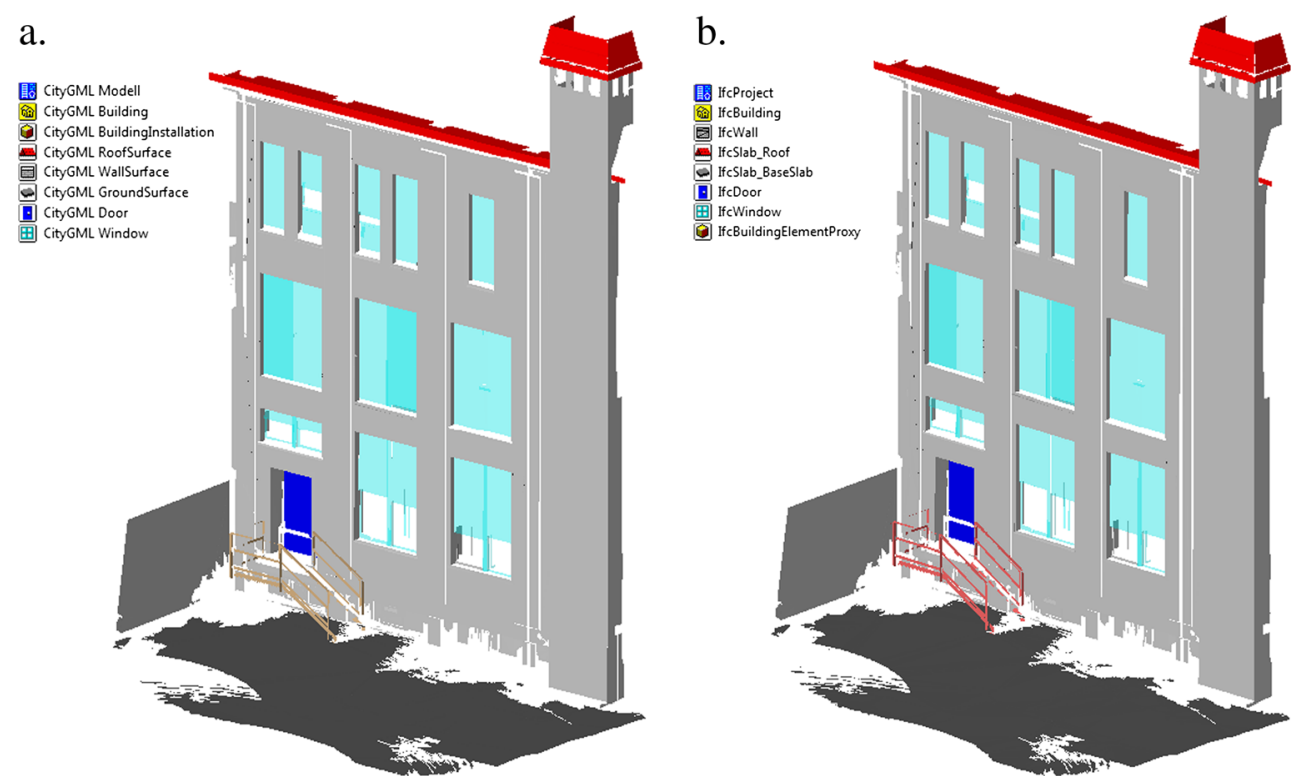
The crucial step for data integration is the co-registration of both datasets (thermographies and façade model) in the same datum. To this end, thermal images are accurately registered by using a bundle adjustment solution combining both thermal and RGB images as described in detail in Previtali et al. (2013).

Here, the orientation of thermal images is performed by using a combined bundle adjustment integrating also a block of RGB images (Scaioni et al. 2012). This solution allows a better control on the quality of results and reduces the number of points to be measured on the thermal images with respect to other traditional approaches. First, only a set of RGB images, acquired with a calibrated camera and fulfilling adequate requirements in terms of overlap and acquisition geometry, is oriented within a standard photogrammetric bundle adjustment. This first adjustment is based on a set of ground control points (GCPs), used to fix the datum, and a set of tie points (TPs). These TPs, whose 3D position is estimated within this first adjustment, are also exploited in a second step for the co-registration of IRT images. This means that TPs should be preferably measured in correspondence of elements that are clearly visible in both RGB and IR images (e.g. window and door corners). Once RGB images have been registered to the reference system of the object model, IR images are added to the block by identifying in these images some TPs among those previously measured in the block of RGB images. Finally, a combined bundle adjustment including both RGB and IR images is computed to work out the exterior orientation (EO) parameters of all images.

\section{Applications and geometrical accuracy assessment}

This section presents the application of the developed modelling procedure to a couple of real building façades. The first one is the previously introduced D'Oggiono Campus building; the second one is a simple building of Politecnico di Milano-Campus Leonardo. In particular, these test are focussed to assess the reliability of the approach under different operational conditions and façade typologies. Quality evaluation is performed for each step of our procedure:
Table 2 Parameters used for façade modelling for the analysed datasets

D’Oggiono Campus building Leonardo Campus building

\begin{tabular}{ll}
\hline RANSAC plane threshold $\varepsilon$ & $1 \mathrm{~cm}$ \\
RANSAC normal threshold $\alpha$ & $20^{\circ}$ \\
Bitmap cell size $\beta$ & $1 \mathrm{~cm}$ \\
RANSAC dominant line threshold $\varepsilon$ & $0.7 \mathrm{~cm}$ \\
\hline
\end{tabular}

are used for evaluating accuracy of automatically detected ones;

- Object calcification: points of each type of façade element are manually selected from the original point clouds and are compared with object classification results.

\section{D’Oggiono Campus building}

This section provides the experiment details of 'D'Oggiono Campus building' where a portion of the Lecco campus headquarter was scanned. The first step of the developed methodology is façade segmentation. The parameters presented in Table 2 were used in the processing.

Starting with the evaluation of the presented façade segmentation algorithm, two different statistics are evaluated. The first one reflects commission that is the probability to erroneously detect a plane, and it is defined as the ratio between the number of wrong detected plane and the total number of real planes. The second one reflects the omission, i.e. the probability of a real plane being undetected, and it is defined as the ratio between undetected planes and the total number of planes. In Table 3, the statistics for both datasets are presented showing good results against both omission and commission errors.

In order to verify the geometrical accuracy of the presented automatic edge detection process, we compared the detected breaklines with the manually derived ground truth model.

Table3 Commission and omission errors for façade segmentation

D’Oggiono Campus Leonardo Campus building building

- Façade segmentation: point clouds of the building façade Automatically detected planes 120 are manually classified into planer clusters and used as ground truth for comparison between reference data and automatic classification results;

- Breaklines identification: the Campus Leonardo building was manually vectorized, starting from the point cloud, Correctly detected planes

116 22

Manually labelled planes

125

Commission error

$3.2 \%$

$7.2 \%$

21

22

Omission error and façade breaklines were identified. Those breaklines 
Table 4 Commission and omission errors for breakline extraction algorithm

\begin{tabular}{lll}
\hline & $\begin{array}{l}\text { D'Oggiono Campus } \\
\text { building }\end{array}$ & $\begin{array}{l}\text { Leonardo Campus } \\
\text { building }\end{array}$ \\
\hline $\begin{array}{l}\text { Automatically extracted } \\
\text { breaklines }\end{array}$ & 505 & 110 \\
$\begin{array}{l}\text { Correctly detected breaklines } \\
\text { Manually derived breaklines }\end{array}$ & 484 & 106 \\
Commission error & $3.58 \%$ & 124 \\
Omission error & $8.68 \%$ & $3.23 \%$ \\
\hline
\end{tabular}

First, the reliability of breakline detection was tested by evaluating commission and omission errors in a way similar to that one previously introduced (Table 4).

The classification performances are evaluated by comparing manually labelled point cloud, used as a reference, with the automatic classification results. Based on the comparison of all façade classes, a confusion matrix is created. The con-fusion matrix together with commission and omission accu-racies is reported in Table 5. In this table, each row represents the detected instances in a class while column represents the actual ones. The commission error reflects the probability that, given an object classified in a certain class, it does not belong to the same class in the reference data. On the other hand, the omission error measure the probability that, given an object in the reference data, it has not been correctly classified. So, commission is a measure of the exactness, whereas recall is a measure by omission. We can observe that the overall classification accuracy, which is calculated as the sum of the correct classified objects divided by the total number, is $97.46 \%$, and omission and commission errors are 0 for five classes.

Finally, the derived model was textured with IRT images. In this case, a small block of three RGB images acquired with a Nikon D700 with a 35-mm lens was used in conjunction

Table5 Confusion matrix for the D’Oggiono Campus building

\begin{tabular}{|c|c|c|c|c|c|c|c|c|c|c|}
\hline \multicolumn{2}{|c|}{ D’Oggiono Campus building } & \multicolumn{9}{|c|}{ Reference } \\
\hline & & Wall & Roof & Sidewall & Window & Door & Ground & Attachment & Total & Commission error $(\%)$ \\
\hline \multirow[t]{9}{*}{ Classified } & Wall & 20 & 0 & 0 & 0 & 0 & 0 & 1 & 21 & 4.7 \\
\hline & Roof & 1 & 6 & 0 & 0 & 0 & 0 & 0 & 7 & 16.7 \\
\hline & Sidewall & 0 & 0 & 40 & 0 & 0 & 0 & 0 & 40 & 0 \\
\hline & Window & 0 & 0 & 0 & 30 & 0 & 0 & 0 & 30 & 0 \\
\hline & Door & 0 & 0 & 0 & 0 & 1 & 0 & 0 & 1 & 0 \\
\hline & Ground & 0 & 0 & 0 & 0 & 0 & 1 & 0 & 1 & 0 \\
\hline & Attachment & 0 & 0 & 0 & 0 & 0 & 0 & 20 & 20 & 0 \\
\hline & Total & 21 & 6 & 40 & 30 & 1 & 1 & 21 & 79 & \\
\hline & Omission error $(\%)$ & 4.7 & 0 & 0 & 0 & 0 & 0 & 5 & & \\
\hline
\end{tabular}

with the IR image (Fig. 13a). Fourteen checkerboard targets, measured with a first-order theodolite, were employed for the registration of laser scans and were also used as GCPs during the first-stage bundle adjustment including the RGB image only. In addition, 20 tie points in correspondence of window and door corners were measured on both RGB and thermal images to obtain the EO parameters of all images (Fig. 13b). Statistics of the combined bundle adjustment showed a final sigma-naught of about 0.9 pixels, which expresses the average measurement precision of points in both datasets. Starting from the 3D textured model (Fig. 13a), thermal and RGB orthophotos (Fig. 20) can also be generated and used in GIS environment for further exhaustive analysis (Previtali et al. 2012).

\section{Campus Leonardo building}

Also, in this second case, the Faro Focus 3D laser scanner was used. Two scans of the building were acquired and fused together after registration. The final point cloud has about five million points and a mean ground sampling distance of $7 \mathrm{~mm}$ (Fig. 14). A quite significant noise is evident in the cloud due to the presence of a large tree just in front of the building. In Table 3, the statistics for segmentation results are presented. As can be seen from Table 4, omission error in the case of Leonardo Campus building is quite large. This is due to the fact (Fig. 15) that the presented algorithm failed in detecting the door edges because it is coplanar with the façade wall.

Then, the accuracy of the reconstructed breaklines was derived by comparing the ground truth position of each line with the position estimated by our algorithm (Fig. 15). In particular, we defined for each edge the absolute modelling error as the absolute magnitude of the difference between the ground truth and model position of the edge vertices. From these measurements, a mean difference of 4 $\mathrm{mm}$ and a standard deviation of $1 \mathrm{~mm}$ were found, confirming the reliability 
a.

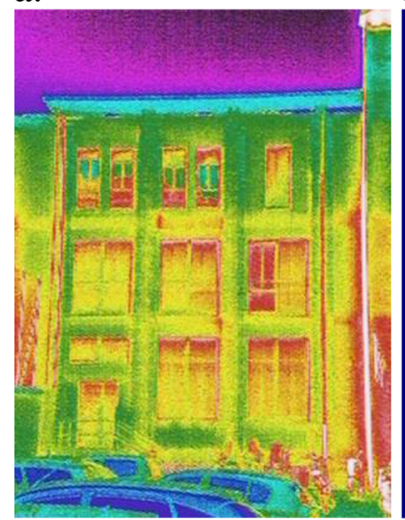

d.

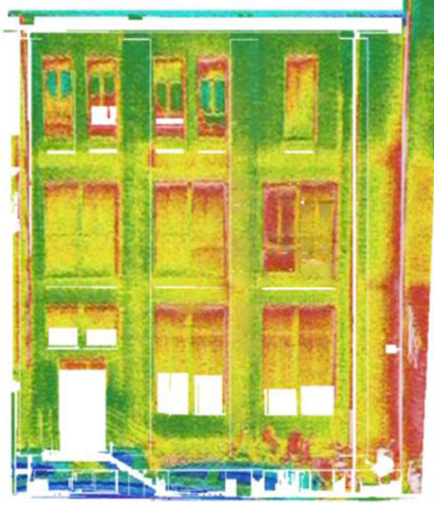

b.

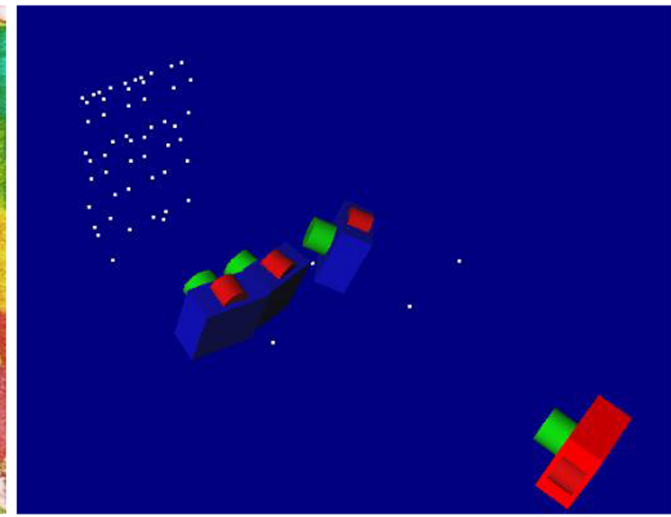

c.

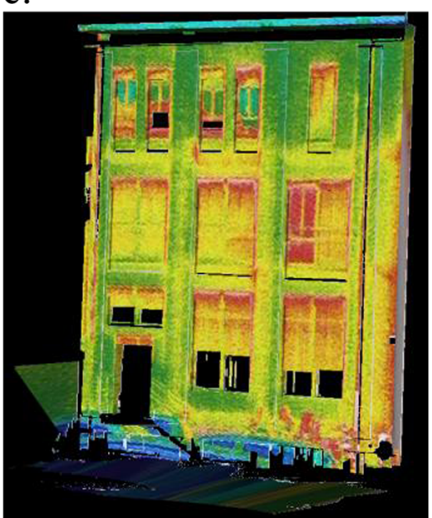

f.

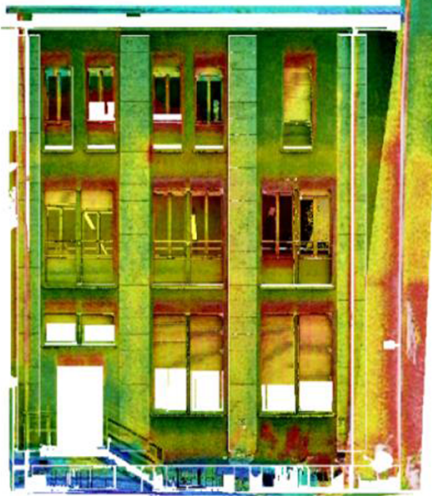

Fig. 13 Thermal image integration for D'Oggiono campus building: the IR image (a), a 3D view showing camera poses of both RGB (blue) and thermal (red) cameras (b), IRT textured façade model (c), IRT orthoimage (d), RGB orthoimage (pixel size $0.9 \mathrm{~mm})(\mathbf{e})$, and thermal pan-sharpened orthoimage (f)

of the matching algorithm which is acceptable if compared to the resolution of the laser scanner.
Finally, the classification performances are evaluated (Table 6). In this case, the overall classification a.

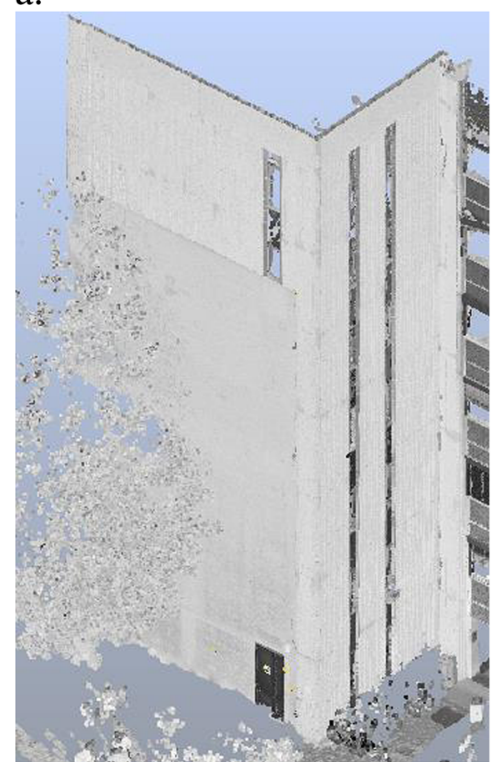

b.

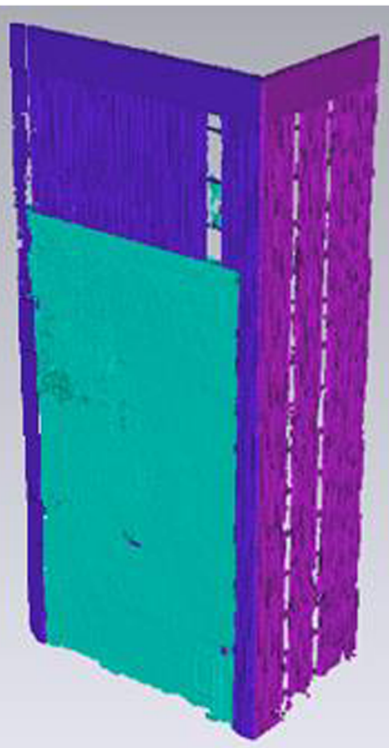

c.

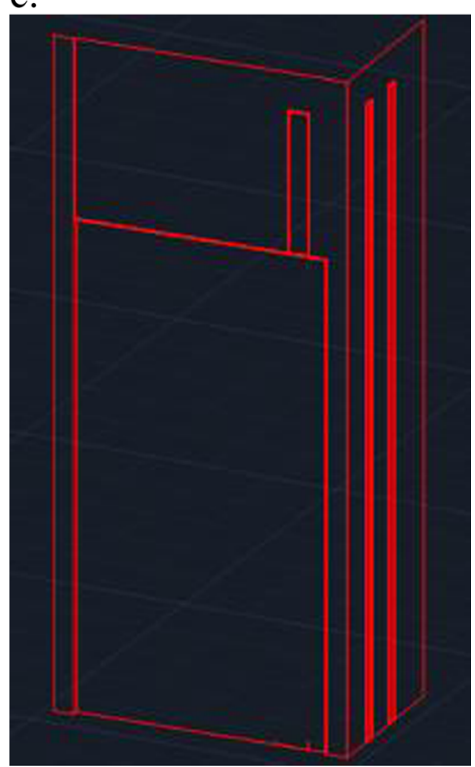

Fig. 14 Test façade: raw point cloud (a), segmented clusters (c), and detected breaklines (c) 
a.

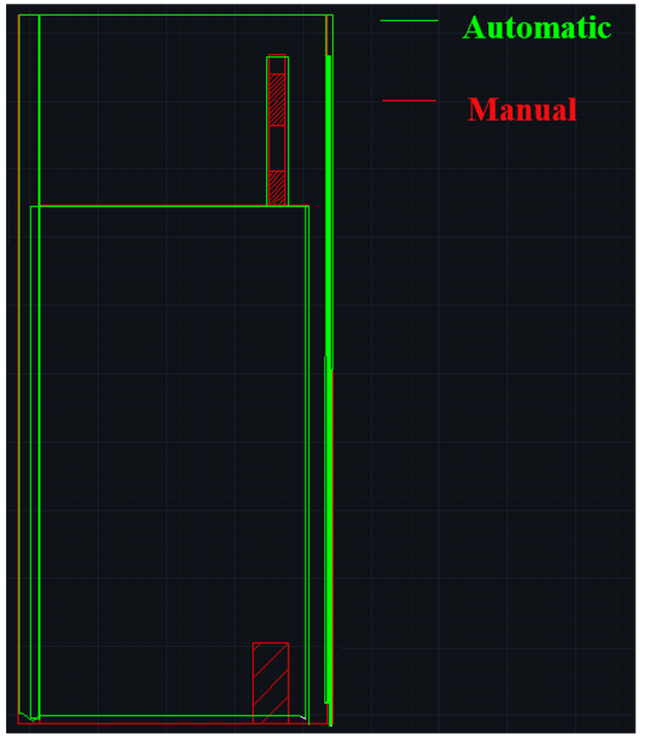

b.

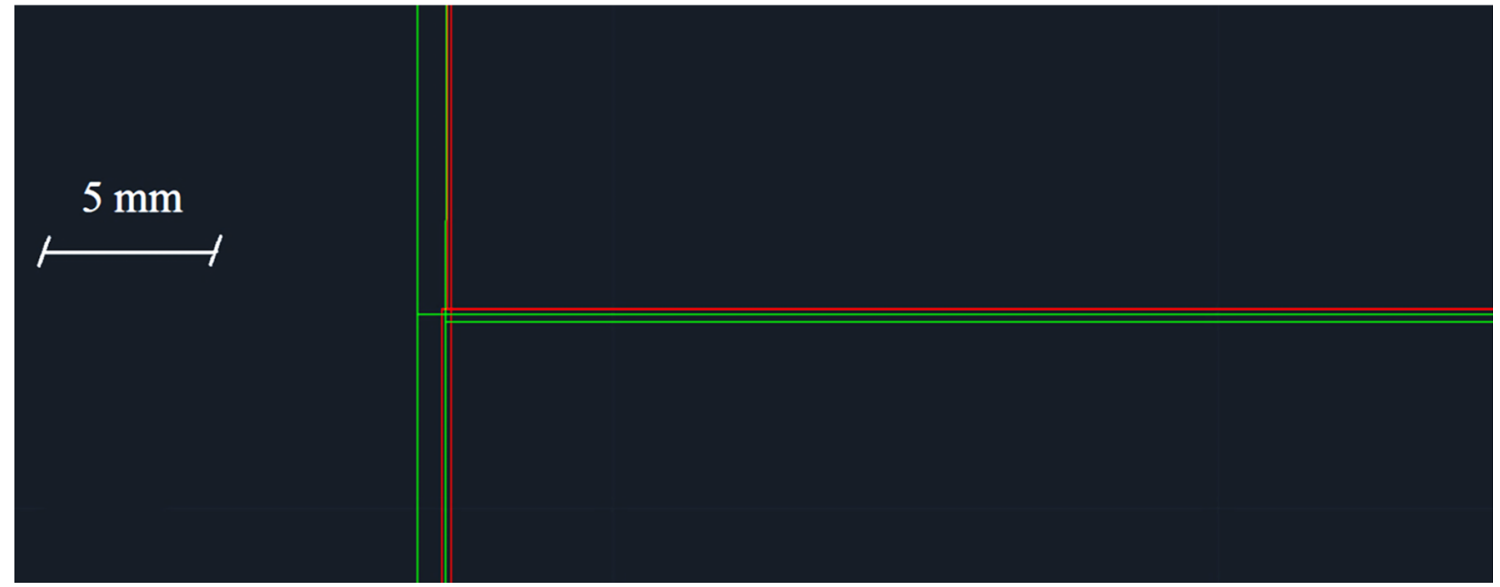

Fig. 15 Test façade: breaklines detected by the automatic (green) and manual (red) procedures (a) and a detail of the comparison

accuracy is $95.45 \%$ while omission and commission This is given by the fact that the only door in the scene errors are 0 for all classes, exception made for door. is coplanar with the façade wall.

Table 6 Confusion matrix for the classification algorithm

\begin{tabular}{|c|c|c|c|c|c|c|c|c|c|c|}
\hline \multicolumn{2}{|c|}{ Leonardo Campus building } & \multicolumn{9}{|c|}{ Reference } \\
\hline & & Wall & Roof & Sidewall & Window & Door & Ground & Attachment & Total & $\begin{array}{l}\text { Commission } \\
\text { error }(\%)\end{array}$ \\
\hline \multirow{9}{*}{ Classified } & Wall & 9 & 0 & 0 & 0 & 1 & 0 & 0 & 10 & 10 \\
\hline & Roof & 0 & 0 & 0 & 0 & 0 & 0 & 0 & 0 & 0 \\
\hline & Sidewall & 0 & 0 & 8 & 0 & 0 & 0 & 0 & 8 & 0 \\
\hline & Window & 0 & 0 & 0 & 3 & 0 & 0 & 0 & 3 & 0 \\
\hline & Door & 0 & 0 & 0 & 0 & 0 & 0 & 0 & 0 & 0 \\
\hline & Ground & 0 & 0 & 0 & 0 & 0 & 1 & 0 & 1 & 0 \\
\hline & Attachment & 0 & 0 & 0 & 0 & 0 & 0 & 0 & 0 & 0 \\
\hline & Total & 9 & 0 & 8 & 3 & 1 & 1 & 0 & 22 & \\
\hline & Omission error $(\%)$ & 0 & 0 & 0 & 0 & 100 & 0 & 0 & & \\
\hline
\end{tabular}




\section{Conclusions}

This paper presented a novel procedure to derive 3D BIM model of building façades starting from unstructured laser scan point clouds and their integration with infrared thermographic analysis. The developed procedure for automatic façade model generation relies on a modified RANSAC approach for planar object identification, which is mainly focussed to avoid badsegmentation results by adding some topological information. Once planar clusters are detected, breaklines of the façade are automatically reconstructed, introducing some priors typical of the architectural style of many modern buildings. A semantic enrichment of the model is obtained by using a classification tree to recognize façade objects and allowing modelling the building at LOD3. Occlusions and data lacks determine some gaps in the automatically generated model. For this reason, the derived models can be used as an alternative to manual CAD models (in the case when only a simplified model is sufficient and then the output of the automatic modelling procedure may suffice) or in conjunction with manually generated models (if very accurate modelling is needed). In the second case, manual modelling can take place after the automatic generation of the model with a consequent increment of the efficiency along with a cost reduction. The proposed methodology was tested on a couple of buildings, and a wider analysis is needed in the future for its validation on a larger number of buildings with different architectural styles. In particular, the segmentation process and the breakline extraction algorithm are not expected featuring criticalities for modern style building where 'Manhattan world assumption' holds. Analyses of other building styles are out of the aims of this paper. On the other hand, the classification strategy may result in significant misclassifications in the case when the analysed building does not feature the priors we have formalized. For example, in our scheme, balconies will be generally categorized as wall attachments while a proper subclass would be more adequate. For future applications, the classification tree will probably need some extensions.

Finally, an innovative solution for the integration of 3D BIM models of complex structures and IR images was presented. In particular, the combined adjustment of thermal images and RGB data can enhance the registration of IR images. The derived BIM model can be used to identify thermal defects and heat losses in order to provide a support for energy retrofitting design at both stages of thermal energy efficiency evaluation and production of executive drawings. In addition, the possibility to integrate BIM functionalities allows one to perform a cost-benefit analysis maximizing the energy saving.

Acknowledgments This work was supported by the EASEE (Envelope Approach to improve Sustainability and Energy efficiency in Existing multi-storey multi-owner residential buildings) project (ID: FP7-2011NMP EeB, EeB.NMP.2011-3 'Energy saving technologies for buildings envelope retrofitting'). Acknowledgements also go to the National Key Basic 973 Research Program of China (Id 2012CB957702).

\section{References}

Africani P, Bitelli G, Lambertinia A, Minghetti A, Paselli E (2013) Integration of LIDAR data into a municipal GIS to study solar radiation. In International Archives of the Photogrammetry, Remote Sensing and Spatial Information Sciences, Volume XL-1/ W1, ISPRS Hannover Workshop 2013, 21 - 24 May 2013, Hannover, Germany, 1-6

Alba M, Barazzetti L, Rosina E, Scaioni M, Previtali M (2011) Mapping infrared data on terrestrial laser scanning 3D models of buildings. Rem Sens 3(9): 1847-1870

Alshawa M, Boulaassal H, Landes T, \& Grussenmeyer P (2009) Acquisition and automatic extraction of facade elements on large sites from a low cost laser mobile mapping system. ISPRS/ 3DARCH09

Alshawabkeh Y, Haala N (2004) Integration of digital photogrammetry and laser scanning for heritage documentation. Int Arch Photogramm Remote Sens Spat Inf Sci 35(Part 5):424-429

Awwad TM, Zhu Q, Du Z, Zhang Y (2010) An improved segmentation approach for planar surfaces from unstructured 3D point clouds. Photogramm Rec 25(129):5-23

Barazzetti L, Erba S, Previtali M, Rosina E, Scaioni M (2013) Mosaicking thermal images of buildings. In SPIE Optical Metrology 2013 (pp. 879108-879108). International Society for Optics and Photonics

Becker S (2009) Generation and application of rules for quality dependent facade reconstruction. ISPRS J Photogramm Remote Sens 64(6): $640-653$

Becker S and Haala N (2007) Refinement of building facades by integrated processing of LIDAR and image data. In PIA07.International Archives of Photogrammetry, Remote Sensing and Spatial Information Sciences (Sept. 2007)

Benner J, Geiger A, Leinemann K (2005) Flexible generation of semantic 3D building models. In: Groeger, G. et al. (Eds.): Proceedings of the 1st International Workshop on Next Generation 3D City Models, Bonn

Boehler W, Heinz G, Marbs A (2002) The potential of non-contact close range laser scanners for cultural heritage recording. Int Arch Photogramm Remote Sens Spat Inf Sci 34.5(C7):430-436

Boulaassal H, Landes T, Grussenmeyer P, Tarsha-Kurdi F (2007) Automatic segmentation of building facades using terrestrial laser data. ISPRS workshop on laser scanning and SilviLaser. Espoo, Finland, pp 65-70

Boulaassal H, Landes T, Grussenmeyer P (2008) Automatic extraction of planar clusters and their contours on building façades recorded by terrestrial laser scanner. VSMM 2008-Conference on Virtual Systems and MultiMedia Dedicated to Digital Heritage. Limassol Cyprus - October 20th - 25th

Boulaassal H, Landes T, Grussenmeyer P (2009) Automatic extraction of planar clusters and their contours on building facades recorded by terrestrial laser scanner. Int J Architect Comput 7(1):1-20

Brumana R, Oreni D, Cuca B, Binda L, Condoleo P, Triggiani M (2013) Strategy for integrated surveying techniques finalized to interpretive models in a byzantine church, Mesopotam, Albania. Accepted on publishing in: Int J Architect Herit, DOI: 10.1080/ 15583058.2011.605202, Elsevier

Cabrelles M, Galcerá S, Navarro S, Lerma J.L, Akasheh T, Haddad, N (2009) Integration of 3D laser scanning, photogrammetry and thermography to record architectural monuments. In Proc. of the 22nd International CIPA Symposium (p. 6)

Comaniciu D, Meer P (2002) Mean shift: a robust approach toward feature space analysis. IEEE Trans Pattern Anal Machine Intell 24: 603-619

Coughlan J.M and Yuille A.L (2001) The Manhattan world assumption: regularities in scene statistics which enable Bayesian inference. $\mathrm{Adv}$ Neural Inform Process Syst, 845-851 
Edis E, Flores-Colen I, de Brito J (2012) Passive thermographic inspection of adhered ceramic claddings: limitations and conditioning factors. J Perform ConstrFacil 1:258

El-Hakim S.F, Beraldin J.A, Picard M, Vettore, A (2003). Effective 3d modeling of heritage sites. In 3-D digital imaging and modeling, 2003. 3DIM 2003. Proceedings. Fourth International Conference on (pp. 302-309). IEEE

Filin S(2004) Surface classification from airborne laser scanning data. Comput Geosci 30 (9-10) (2004) 1033-1041

Fishler MA, Bolles RC (1981) Random sample consensus: a paradigm for model fitting with application to image analysis and automated cartography. Commun of the AMC 24(6):381-395

Fox S, Hietanen J (2007) Interorganizational use of building information models: potential for automational, informational and transformational effects. Constr Manag Econ 25.3(2007):289-296

Geibel R, Stilla U (2000) Segmentation od laser altimeter data for building reconstruction: different procedures and comparison. Proc Internalional Arch Photogramm Remote Sens, Amst XXXIII(Part B3):326-334

González-Jorge H, Lagüela S, Krelling P, Armesto J, MartínezSánchez J (2012) Single image rectification of thermal images for geometric studies in façade inspections. Infrared Phys Technol 55(5):421-426

Gröger G, Plümer L (2012) CityGML-Interoperable semantic 3D city models. ISPRS J Photogramm Remote Sens 71:12-33

Hallberg D and Tarandi V (2009) On the use of 4D BIM in LMS for construction works. J Inf Technol in Construction (Itcon)

Hansard M., Lee S., Choi O., Horaud R. (2013). Time-of-flight cameras. Springer

Hartley R, Ziesserman A (2003) Multiple view geometry in computer vision. Second edition. Cambridge University Press, Cambridge, $p p$ $117-121,655$ pages

Hesami R, Hossein-Nezhad R, Bab-Hadiashar A (2010) Range segmentation of large building exteriors: a hierarchical robust approach. Comput Vis Image Understand 114(4):475-490

Hough P.V.C(1962) Method and means for recognizing complex patterns. U.S. Patent $3,069,654$

Isikdag U, Zlatanova S (2009) Towards defining a framework for automatic generation of buildings in CityGML using BIM. In: Lee J, Zlatanova S (eds) 3D Geo-information Sciences. Springer-Verlag, LNG\&C, pp 79-96

Jenke P, Krückeberg B, Strasser W (2008) Surface reconstruction from fitted shape primitives. In 13th International fall workshop vision, modeling and visualization, pages $31-40$

Lagüela S, González-Jorge H, Armesto J, Arias P (2011) Calibration and verification ofthermographic cameras for geometric measurements, Infrared Phys. Technol 54:92-99

Lerma J.L and Biosca J.M(2005) Segmentation and filtering of laser scanner data for cultural heritage. CIPA $2005 \mathrm{XX}$ International Symposium, 26 September - 01 October, 2005, Torino, Italy

Lerones PM, Fernández JL, Gil ÁM, Gómez-García-Bermejo J, Casanova EZ (2010) A practical approach to making accurate 3D layouts of interesting cultural heritage sites through digital models. J Cult Herit 11(1):1-9

Lubowiecka I, Armesto J, Arias P, Lorenzo H (2009) Historic bridge modelling using laser scanning, ground penetrating radar and finite element methods in the context of structural dynamics. Eng Struct 31(11):2667-2676

Luhmann T, Ohm J, PiechelJ, Roelfs T (2010) Geometric calibration of thermographic cameras, International Archives of Photogrammetry, Remote Sensing and Spatial Information Sciences, Newcastle upon Tyne, UK, Vol. XXXVIII, Part 5, pp. 411-416

Luhmann T, Robson S, Kyle S, Harley I (2011) Close range photogrammetry: principles, techniques and applications. Whittles Publishing, Dunbeath, Caithness, Scotland, 528 pages
Martínez J, Soria-Medina A, Arias P, Buffara-Antunes AF (2012) Automatic processing of terrestrial laser scanning data of building façades. Autom Construc 22:298-305

Masaharu H and Hasegawa H.(2000) Three-dimensional city modelling from laser scanner data by extracting building polygons using region segmentation method, Proc. International Archives of Photogrammetry and Remote Sensing, Amsterdam

Menna F, Remondino F, Battisti R, Nocerino E(2011) Geometric investigation of a gaming active device. In: Int. Conf. 'Videometrics, Range Imaging, and Applications XI,' 23-26 May 2011, Munich, Germany (Proc. of SPIE, Vol. 8085, No. 80850G), 15 pages (e-doc)

Moreira J. M, Nex F, Agugiaro G, Remondino F, Lima N. J (2013) From DSM to 3D building models: a quantitative evaluation, In International Archives of the Photogrammetry, Remote Sensing and Spatial Information Sciences, Volume XL-1/W1, ISPRS Hannover Workshop 2013, 21 - 24 May 2013, Hannover, Germany, 213-219

Murphy M, McGovern E, Pavia S (2009) Historic building information modelling (HBIM). Struct Surv 27(4):311-327

Musialski P, Wonka P, Aliaga D.G, Wimmer M., van Gool L, Purgathofer W(2012) A survey of urban reconstruction. In EUROGRAPHICS 2012 State of the Art Reports

Naci Y (2007) Documentation of cultural heritage using digital photogrammetry and laser scanning. J Cult Herit 8(4):423-427

Nagel C, Stadler A, Kolbe T (2009) Conceptual requirements for the automatic reconstruction of building information models from uninterpreted 3D models. Academic Track of Geoweb 2009 Conference, Vancouver

Nan L, Sharf A, Zhang H, Cohen ORD, Chen B (2010) SmartBoxes for interactive urban reconstruction. ACMTransactions on Graphics 29, 4 (July 2010), 1.17,19

Oreni D, Cuca B, Brumana R (2012a) Three dimensional virtual models for better comprehension of architectural heritage construction techniques and its maintenance over time. In: Ioannides M, Fritsch D, Remondino F (eds) Progress in cultural heritage. Lecture Notes in Computer Science, vol. 7616. Springer, Heidelberg, London, pp 533-542

Oreni D, Brumana R, Cuca B (2012b) Towards a methodology for 3D content models. The reconstruction of ancient vaults for maintenance and structural behaviour in the logic of BIM management. In: Ga-brieleGuidi, Addison AC (eds) Virtual systems in the information society. IEEE, NJ, pp 475-482

Previtali M, Erba S, Rosina E, Redaelli V, Scaioni M, Barazzetti L (2012) Generation of a GIS-based environment for infrared thermography analysis of buildings. In SPIE Optical Engineering + Applications (pp. 85110U-85110U). International Society for Optics and Photonics

Previtali M, Barazzetti L, Redaelli V, Scaioni M, Rosina E (2013) Rigorous procedure for mapping thermal infrared images on threedimensional models of building façades. J Appl Remote Sens 7(1): 073503-073503

$\mathrm{Pu} \mathrm{S}$ and Vosselman G (2006) Automatic extraction of building features from terrestrial laser scanning. IAPRS, vol. 36, part 5, Dresden, Germany, September 25-27, 5 p. (on CD-ROM)s

$\mathrm{Pu}$ S, Vosselman G (2009) Knowledge based reconstruction of building models from terrestrial laser scanning data. ISPRS J Photogramm Remote Sens 64(6):575-584

Ripperda N and Brenner C (2006) Reconstruction of facade structures using a formal grammar and rjMCMC. Pattern Recognition, Ed. by Franke, K., et al., Springer, pp. 750-759

Sapkota P.P (2008) Segmentation of coloured point cloud data. M.Sc. thesis, ITC, Enschede, the Netherlands. 67 pages

Scaioni M, Rosina E, Barazzetti L, Previtali M, Redaelli V, (2012) Highresolution texturing of building facades with thermal images. Proc. of SPIE Defence, Security and Sensing, Vol. 8354, 23-27 April, Baltimore, USA 
Schnabel R, Wahl R, Klein R(2007) Efficient RANSAC for point-cloud shape detection. In Computer graphics forum, Vol. 26, No. 2, Blackwell Publishing, June 2007, pages 214-226

Stamos I, Yu G, Wolberg G, Zokai S (2006) 3D modeling using planar segments and mesh elements. 3rd International Symposium on 3D Data Processing, Visualization\& Transmission, University of North Carolina, Chapel Hill, June 14-16 2006

Stewart CV (1997) Bias in robust estimation caused by discontinuities and multiple structures. IEEE Trans Pattern Anal Mach Intell 19: 818-833

Tarsha-Kurdi F, Landes T, Grussenmeyer P (2008) Extended RANSAC algorithm for automatic detectionof building roof planes from Lidar data. Photogramm J Finl 21(1):97-109
Vanegas C.A, Aliaga D.G, Benes B (2012) Automatic extraction of Manhattan-World building masses from 3D laser range scans. IEEE transactions on visualization and computer graphics, 99

Yastikli N and Guler E (2013) Performance evaluation of thermographic cameras for photogrammetric measurements. In International Archives of the Photogrammetry, Remote Sensing and Spatial Information Sciences, Volume XL-1/W1, ISPRS Hannover Workshop 2013, 21 - 24 May 2013, Hannover, Germany, 383-387

Zheng Q, Sharf A, Wan G, Li Y, Mitra N.J, Cohen-O.R.D. Chen B(2010) Non-local scan consolidation for 3D urban scenes. ACM Transactions on Graphics 29, 4 (July 2010), 1. 11 